\title{
Coexistence of regular and irregular dynamics in complex networks of pulse-coupled oscillators
}

\author{
Marc Timme, Fred Wolf, and Theo Geisel \\ Max-Planck-Institut für Strömungsforschung, 37073 Göttingen, Germany
}

\begin{abstract}
For general networks of pulse-coupled oscillators, including regular, random, and more complex networks, we develop an exact stability analysis of synchronous states. As opposed to conventional stability analysis, here stability is determined by a multitude of linear operators. We treat this multioperator problem exactly and show that for inhibitory interactions the synchronous state is stable, independent of the parameters and the network connectivity. In randomly connected networks with strong interactions this synchronous state, displaying regular dynamics, coexists with a balanced state that exhibits irregular dynamics such that external signals may switch the network between qualitatively distinct states.

PACS numbers: 89.75.-k, 05.45.-a, 87.10.+e
\end{abstract}

Complex networks appear as a variety of natural and artificial systems, ranging from the world wide web and electrical power grids to metabolic and neural networks [1, 2]. While recent studies have focussed on their structure [1], the dynamics in such networks constitute a challenging issue of current and future research [2]. Even if the individual vertices of the network are simple dynamical systems, such as limit cycle oscillators, an exact mathematical analysis of their collective dynamics is often highly intricate, due to the complex connectivity structure.

As a prototypical class of dynamical systems interacting on networks, pulse-coupled units have received a significant amount of interest because of their relevance to diverse natural systems [3, 4, [5, 6, 7] including cardiac pacemaker cells, flashing fireflies, earthquakes, and biological neural networks. Particularly in neuroscience, these models [8] are essential for understanding collective dynamic phenomena such as synchronization or the propagation of sensory signals through extended networks $[9,10]$. Although biological neural networks, like other networks occurring in the real world, often possess a complex connectivity structure, most theoretical studies on pulse-coupled oscillators are either restricted to networks of globally coupled oscillators and simple regular networks, or work in some mean field limit [3, 4, 5, 6].

In this Letter, we study pulse-coupled oscillators interacting on networks with general connectivities, including fully connected, regular, random, and more complex topologies. We develop an exact stability analysis of the synchronous state. In contrast to conventional stability problems, the first order stability operator here is not linear, but can be expressed by a multitude of distinct linear operators, the domains of which depend on the rank order of a specific perturbation. For generally structured networks, the number of operators increases exponentially with the size of the network. Our analysis provides a method to treat this multi-operator problem analytically. For networks with inhibitory couplings, we prove that the synchronous state is stable, independent of the parameters and the connectivity structure.

Proceeding from this result, we show that the synchronous state, that displays regular dynamics, coexists with a state of highly irregular dynamics in randomly connected networks. We suggest a simple mechanism for switching between these states. These results establish that the behavior of networks of pulse-coupled units for a given set of parameters may be dominated by qualitatively distinct dynamical states.

We consider a system of $N$ coupled oscillators [3] which interact on directed graphs by sending and receiving pulses. The structure of this graph is specified by the sets $\operatorname{Pre}(i)$ of presynaptic oscillators that send pulses to oscillator $i$. These sets determine the sets Post $(i)$ of postsynaptic oscillators that receive pulses from $i$. A phaselike variable $\phi_{i}(t) \in(-\infty, 1]$ specifies the state of each oscillator at time $t$. The dynamics of a non-interacting oscillator $i$ is given by

$$
d \phi_{i}(t) / d t=1 .
$$

When oscillator $i$ reaches a threshold, $\phi_{i}(t)=1$, its phase is reset to zero, $\phi_{i}\left(t^{+}\right)=0$, and the oscillator is said to "fire". A pulse is sent to all postsynaptic oscillators $j \in \operatorname{Post}(i)$ which receive this signal after a delay time $\tau$. Depending on whether the input is subthreshold or suprathreshold, the incoming signal induces a phase jump

$$
\phi_{j}\left((t+\tau)^{+}\right):=\min \left\{U^{-1}\left(U\left(\phi_{j}(t+\tau)\right)+\varepsilon_{j i}\right), 1\right\}
$$

which depends on the instantaneous phase $\phi_{j}(t+\tau)$ of the postsynaptic oscillator and the coupling strength $\varepsilon_{j i}$. The phase dependence is determined by a twice continuously differentiable 'potential' function $U(\phi)$ that is assumed to be strictly increasing, $U^{\prime}(\phi)>0$, concave (down), $U^{\prime \prime}(\phi)<0$, and normalized such that $U(0)=0$, $U(1)=1$ (cf. [3]).

By choosing an appropriate function $U$, this model is equivalent (cf. 11]) to different well known models of interacting threshold elements. For instance, for the leaky 
integrate-and-fire oscillator defined by the linear differential equation $\dot{V}=I-V$ [8] (and threshold at $V=1$ ), one obtains $U_{\mathrm{IF}}(\phi)=I\left(1-e^{-T_{\mathrm{IF}} \phi}\right)$ where $T_{\mathrm{IF}}=\log (I /(I-1))$ is the period of a non-interacting oscillator and $I>1$ is a suprathreshold external current. Oscillators described by nonlinear differential equations are covered by the Mirollo-Strogatz approach, too. For instance, the conductance based threshold model of a neuron [8] leads to a different, more complicated function $U_{\mathrm{CB}}(\phi)$ (for details see [1]]). All analytical results presented here are derived for the above, general class of interaction functions. In numerical investigations, we use the functional form $U_{\mathrm{IF}}$ but find qualitatively similar results for different $U$. In this Letter, we focus on inhibitorily coupled networks (all $\varepsilon_{i j} \leq 0$ and $\varepsilon_{i i}=0$ ).

We perform a stability analysis of the synchronous state $\left(\phi_{i}(t)=\phi_{0}(t)\right.$ for all $\left.i\right)$ that exists if the coupling strengths are normalized such that $\sum_{j \in \operatorname{Pre}(i)} \varepsilon_{i j}=\varepsilon<0$. Its period is given by

$$
T=\tau+1-\beta_{0}
$$

where $\beta_{0}=U^{-1}(U(\tau)+\varepsilon)$. To construct a stroboscopic map, a perturbation $\boldsymbol{\delta}(0) \equiv \boldsymbol{\delta}=\left(\delta_{1}, \ldots, \delta_{N}\right)$ of the phases, defined by

$$
\phi_{i}(0)=\phi_{0}(0)+\delta_{i},
$$

is ordered according to the rank order $\operatorname{rank}(\boldsymbol{\delta})$ of the $\delta_{i}$ : For each oscillator $i$ we label the perturbations $\delta_{j}$ of its presynaptic oscillators $j$ (for which $\varepsilon_{i j} \neq 0$ ) according to their size

$$
\Delta_{i, 1} \geq \Delta_{i, 2} \geq \ldots \geq \Delta_{i, k_{i}}
$$

where $k_{i}:=|\operatorname{Pre}(i)|$ is the number of its presynaptic oscillators, called in-degree in graph theory [12]. In addition, we define $\Delta_{i, 0}=\delta_{i}$. For illustration assume that an oscillator $i$ has exactly two presynaptic oscillators $j_{1}$ and $j_{2}$ such that $\operatorname{Pre}(i)=\left\{j_{1}, j_{2}\right\}$ and $k_{i}=2$. For certain perturbations, oscillator $i$ first receives a signal from oscillator $j_{2}$ and slightly later from oscillator $j_{1}$. This determines the rank order $\left(\delta_{j_{2}}>\delta_{j_{1}}\right)$ such that $\Delta_{i, 1}=\delta_{j_{2}}$ and $\Delta_{i, 2}=\delta_{j_{1}}$.

Using the phase shift function $h(\phi, \varepsilon):=U^{-1}(U(\phi)+\varepsilon)$ and denoting $D_{i, n}:=\Delta_{i, n-1}-\Delta_{i, n}$ for $n \in\left\{1, \ldots, k_{i}\right\}$ we compute the time evolution of phase-perturbations $\delta_{i} \ll$ 1 , starting near $\phi_{0}(0)=\tau / 2$ without loss of generality. The stroboscopic time- $T$ map of the perturbations, $\delta_{i} \mapsto$ $\delta_{i}(T)$, is obtained from the scheme

\begin{tabular}{|c|c|}
$t$ & $\phi_{i}(t)$ \\
\hline 0 & $\frac{\tau}{2}+\delta_{i}=: \frac{\tau}{2}+\Delta_{i, 0}$ \\
$\frac{\tau}{2}-\Delta_{i, 1}$ & $h\left(\tau+D_{i, 1}, \varepsilon_{i j_{1}}\right)=: \beta_{i, 1}$ \\
$\frac{\tau}{2}-\Delta_{i, 2}$ & $h\left(\beta_{i, 1}+D_{i, 2}, \varepsilon_{i j_{2}}\right)=: \beta_{i, 2}$ \\
$\vdots$ & $\vdots$ \\
$\frac{\tau}{2}-\Delta_{i, k_{i}}$ & $h\left(\beta_{i, k_{i}-1}+D_{i, k_{i}}, \varepsilon_{i j_{k_{i}}}\right)=: \beta_{i, k_{i}}$ \\
$\frac{\tau}{2}-\Delta_{i, k_{i}}$ & reset: $1 \mapsto 0$ \\
$+1-\beta_{i, k_{i}}$ &
\end{tabular}

where the right column gives the phases $\phi_{i}(t)$ of oscillator $i$ at times $t$ of pulse receptions or reset given in the left column. Here the presynaptic oscillator from which oscillator $i$ receives the $n^{\text {th }}$ pulse during this cycle is labeled by $j_{n}$. The time to threshold

$$
T_{i}^{(0)}:=\frac{\tau}{2}-\Delta_{i, k_{i}}+1-\beta_{i, k_{i}}
$$

is always smaller than the period $T$. Hence the period- $T$ map of the perturbation can be expressed as

$$
\delta_{i}(T)=T-T_{i}^{(0)}-\frac{\tau}{2}=\beta_{i, k_{i}}-\beta_{0}+\Delta_{i, k_{i}}
$$

Expanding $\beta_{i, k_{i}}$ for small $D_{i, n}$ one can prove by induction that to first order

$$
\beta_{i, k_{i}} \doteq \beta_{0}+\sum_{n=1}^{k_{i}} p_{i, n-1} D_{i, n}
$$

where

$$
p_{i, n}:=\frac{U^{\prime}\left(U^{-1}\left(U(\tau)+\sum_{m=1}^{n} \varepsilon_{i j_{m}}\right)\right)}{U^{\prime}\left(U^{-1}(U(\tau)+\varepsilon)\right)}
$$

for $n \in\left\{0,1, \ldots, k_{i}\right\}$. This results in a first order map

$$
\boldsymbol{\delta}(T) \doteq A \boldsymbol{\delta}
$$

where the elements of the matrix $A$ are given by

$$
A_{i j}= \begin{cases}p_{i, n}-p_{i, n-1} & \text { if } j=j_{n} \in \operatorname{Pre}(i) \\ p_{i, 0} & \text { if } j=i \\ 0 & \text { if } j \notin \operatorname{Pre}(i) \cup\{i\} .\end{cases}
$$

Since $j_{n}$ in (9) and (11) identifies the $n^{\text {th }}$ pulse received during this cycle by oscillator $i$, the first order operator depends on the rank order of the perturbations, $A=A(\operatorname{rank}(\boldsymbol{\delta}))$, and the map $A \boldsymbol{\delta}$ is piecewise linear. In general, signals arriving almost simultaneously at the same oscillator induce different phase changes, depending on the order of arrival: For the above example of an oscillator $i$ with exactly two presynaptic oscillators $j_{1}$ and $j_{2}$ and equal coupling strengths, $\varepsilon_{i, j_{1}}=\varepsilon_{i, j_{2}}$, the first of the two arriving signals has a larger effect, encoded in the $p_{i, n}$, than the second, by virtue of the concavity of $U(\phi)$ (cf. Eq. (2) ). The respective matrix elements $A_{i, j_{1}}$ and $A_{i, j_{2}}$ are differences between certain $p_{i, n}$ and therefore have different values depending on which signal is received first. This is induced by the structure of the network together with the jump-like interactions. For networks with homogeneous, global coupling different matrices $A$ can be identified by appropriately permuting the oscillator indices. In general, however, this is impossible.

Hence, in this stability problem, given a network structure, one generally has to deal with an exponential number of operators instead of a single stability matrix. We 
treat all these operators simultaneously: It is straightforward to show that for all matrices $A$ (independent of the rank order of a perturbation and the parameters) the matrix elements are non-negative, $A_{i j} \geq 0$. Due to time-translation invariance all $A$ are normalized rowwise, $\sum_{j} A_{i j}=1$ for all $i$, and exhibit a trivial eigenvalue $\lambda_{1}=1$. Moreover, the diagonal elements are identical and smaller than one, $A_{i i}=A_{0}<1$. The synchronous state is thus stable, because the inequality

$$
\begin{aligned}
\max _{i}\left|\delta_{i}(T)\right| & \leq \max _{i} \sum_{j}\left|A_{i j}\right|\left|\delta_{j}\right| \\
& \leq \max _{i} \sum_{j}\left|A_{i j}\right| \max _{k}\left|\delta_{k}\right|=\max _{k}\left|\delta_{k}\right|
\end{aligned}
$$

is satisfied for all matrices $A$.

For a convex potential function $U$, where $U^{\prime \prime}>0$, and excitatory interactions $\left(\varepsilon_{i j} \geq 0\right)$ the synchronous state is stable as well. The above proof applies if the total input $\varepsilon$ is not suprathreshold, i.e. $\varepsilon<1-U(\tau)$. Thus whereas excitatory interactions must not be too strong for applicability of the proof, inhibititory interactions may be arbitrarily strong.

For structural stability of the stable synchronous state it is required that the non-trivial eigenvalues of the matrices $A$ are separated from the unit circle. An instructive example is given by a network of integrate-and-fire oscillators, $U(\phi)=U_{\mathrm{IF}}(\phi)$, where all matrices (11) are degenerate if $\varepsilon_{i j}=\varepsilon / k_{i}$ for all $j \in \operatorname{Pre}(i)$. In this case, the eigenvalues of a single matrix completely characterize the dynamics in the vicinity of the synchronous state. Numerically, we find that in large random networks in which every connection is present with probability $p$ all non-trivial eigenvalues are located in a disk $D=\left\{z \in \mathbb{C}|| z-A_{0} \mid \leq r\right\}$ of radius $r$ that is centered at $A_{0}<1$ and separated from the unit circle. An estimate for the radius, $r=\left(1-A_{0}\right)\left(p^{-1}-1\right)^{1 / 2} N^{-1 / 2}$ for $N \gg 1$, can be obtained from the theory of Gaussian asymmetric random matrices 13 . We find that this estimate well agrees with our numerical results [14]. This indicates that in the limit of large $N$, all non-trivial eigenvalues are concentrated near $z=A_{0}$ and thus separated from unit circle.

The above analysis shows that for inhibitory coupling the synchronous state is stable, independent of the parameters and the network structure. Numerical simulations show that for a network at given parameters this synchronous state often coexists with one or more other attractors. A particularly important example which occurs in randomly connected networks with strong interactions, is a balanced state (cf. [5, 15]) that exhibits irregular dynamics. In this balanced state, found originally in binary neural networks [15], inhibitory and excitatory inputs cancel each other on average but fluctuations lead to a variability of the membrane potential and a high irregularity in firing times (see also [5]). Figures [1a,b display sample trajectories of the potentials $U\left(\phi_{i}\right)$ of three oscil-
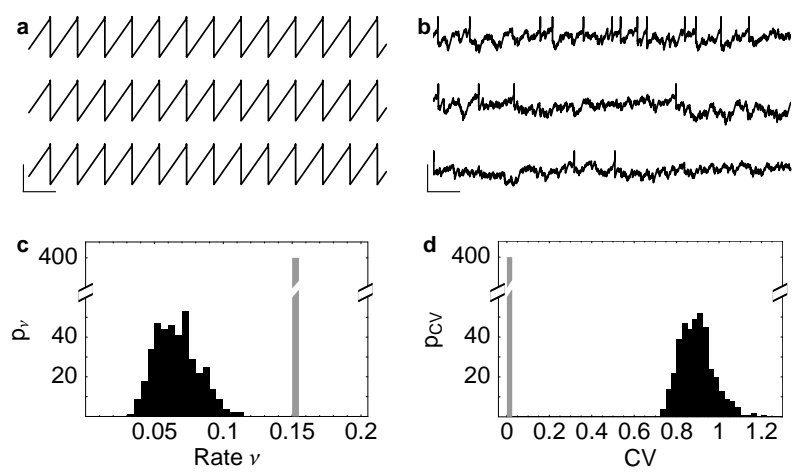

FIG. 1: Coexistence of (a) synchronous and (b) irregular dynamics in a random network $(N=400, p=0.2, I=4.0$, $\varepsilon=16.0, \tau=0.035)$. (a),(b): Trajectories of the potential $U\left(\phi_{i}\right)$ of three oscillators (angular bars: time scale (horizontal) $\Delta t=8$; potential scales (vertical) (a) $\Delta U=8$, (b) $\Delta U=2$; spikes of height $\Delta U=1$ added at firing times). (c),(d): Distributions (c) $p_{\nu}$ of rates and (d) $p_{\mathrm{CV}}$ of the coefficient of variation, displayed for the irregular (dark gray) and synchronous (light gray) dynamics.

lators for the same random network, making obvious the two distinct kinds of coexisting dynamics.

The dynamical differences are quantified by a histogram $p_{\nu}$ of oscillator rates (Fig. 1)

$$
\nu_{i}=\left(\left\langle t_{i, n+1}-t_{i, n}\right\rangle_{n}\right)^{-1}
$$

the reciprocal values of the time averaged inter-spikeintervals. Here the $t_{i, n}$ are the times when oscillator $i$ fires the $n^{\text {th }}$ time. The temporal irregularity of the firing-sequence of single oscillators $i$ is measured by the coefficient of variation

$$
C V_{i}=\left(\nu_{i}^{2}\left\langle\left(t_{i, n+1}-t_{i, n}\right)^{2}\right\rangle_{n}-1\right)^{\frac{1}{2}},
$$

defined as the ratio of the standard deviation of the interspike intervals and their average. A histogram $p_{\mathrm{CV}}$ of the $C V_{i}$ (Fig. 11d) shows that the irregular state exhibits coefficients of variation near one, the coefficient of variation of a Poisson process. Such irregular states occur robustly when changing parameters and network topology; on the other hand, the size of the basin of attraction of the synchronous state is also significant and increases with increasing delay $\tau$.

The coexistence of two qualitatively different kinds of dynamics leads to the question how regular dynamics can be induced when the system currently is in an irregular state and vice versa. A simple mechanism to synchronize oscillators that are in a state of irregular firing is the delivery of two sufficiently strong external excitatory (phase-advancing) pulses that are separated by a time $\Delta t \in(\tau, 1)$, cf. Fig. 2] The first pulse then leads to a synchronization of phases due to simultaneous suprathreshold input (cf. Eq. (2)) . If there are traveling signals that have been sent but not received at the time 


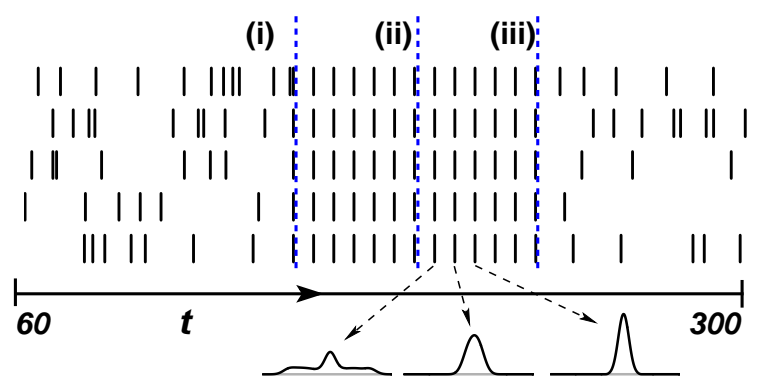

FIG. 2: Switching between synchronous and irregular dynam$\operatorname{ics}(N=400, p=0.2, I=4.0, \varepsilon=16.0, \tau=0.14)$. Firing times of five oscillators are shown in a time window $\Delta t=240$. Vertical dashed lines mark external perturbations: (i) large excitatory pulses lead to synchronous state, (ii) a small random perturbation $\left(\left|\Delta \phi_{i}\right| \leq 0.18\right)$ is restored (iii) a sufficiently large random perturbation $\left(\left|\Delta \phi_{i}\right| \leq 0.36\right)$ leads to an irregular state. Bottom: Time evolution of the spread of the spike times after perturbation (ii), total length $\Delta t=0.25$ each.

of the first pulse, a second pulse after a time $\Delta t>\tau$ is needed that synchronizes the phases after all internal signals have been received. This synchronous state is not affected by small random perturbations, whereas large random perturbations lead back to irregular dynamics (Fig. 2). Mechanisms for both directions of switching may be realized in biological neural networks by external neuronal populations: While strong external pulses may be generated by external neurons that are highly synchronized, a random perturbation can be realized by neurons which fire irregularly.

Most previous studies of the dynamics of networks of pulse-coupled units focussed on regular networks or worked in some mean field limit $[3,44,5,6]$. These studies often relied on the analysis of bifurcations from one state to another as an external parameter is changed. Based on the stability analysis developed here, that applies to networks with general connectivity, we have demonstrated that regular synchronous dynamics may coexist with irregular dynamics in sufficiently complex networks. The coexistence of qualitatively different states at identical parameters indicates that bifurcation approaches may often not give a complete picture of the network dynamics, if the network structure is too complex. This fact may well apply not only to networks of pulse-coupled units but also to the dynamics of many other complex networks. In addition, our results emphasize that in complex networks of pulse-coupled units the occurrence of temporally regular and irregular firing patterns may typically reflect the collective state of the network rather than the dynamics of individual units.

The analysis presented in this Letter demonstrates that the dynamics in certain complex networks can be revealed by considering the vertices as units with simple dynamical properties, e.g. intrinsic oscillators. Such systems provide promising starting points for future studies addressing the dynamics in networks, now that important aspects of their complex structure have been understood [1].

We thank M. Diesmann, D. Hansel, M. Holicki, H. Sompolinsky, and C. van Vreeswijk for helpful comments.

[1] S. Bornholdt and H. G. Schuster (eds.), Handbook of Graphs and Networks, (Wiley-VCH, Weinheim, 2002); R. Albert and A.-L. Barabási, Rev. Mod. Phys. 74, 47 (2002); S. N. Dorogovtsev and J. F. F. Mendes, Adv. Phys. 51, 1079 (2002).

[2] S. H. Strogatz, Nature 410, 268 (2001).

[3] R. E. Mirollo and S. H. Strogatz, SIAM J. Appl. Math. 50, 1645 (1990); U. Ernst, K. Pawelzik, and T. Geisel, Phys. Rev. Lett. 74, 1570 (1995); Phys. Rev. E 57, 2150 (1998).

[4] L. F. Abbott and C. van Vreeswijk, Phys. Rev. E 48, 1483 (1993); A. V. M. Herz and J. J. Hopfield, Phys. Rev. Lett. 75, 1222 (1995); M. V. Tsodyks, I. Mitkov, and H. Sompolinsky, Phys. Rev. Lett. 71, 1280 (1993); Á. Corral, C. J. Pérez, A. Díaz-Guilera, and A. Arenas, Phys. Rev. Lett. 75, 3697 (1995); W. Gerstner, J. L. van Hemmen, and J. D. Cowan, Neural Comp. 8, 1653 (1996); C. v. Vreeswijk, Phys. Rev. E 54, 5522 (1996); P. C. Bressloff, S. Coombes, and B. de Souza, Phys. Rev. Lett. 79, 2791 (1997); D. Golomb and D. Hansel, Neural Comp. 12, 1095 (2000); C. van Vreeswijk, Phys. Rev. Lett. 84, 5110 (2000). D. Hansel and G. Mato, Phys. Rev. Lett. 86, 4175 (2001).

[5] N. Brunel and V. Hakim, Neural Comp. 11, 1621 (1999).

[6] R. O. Dror et al., Biol. Cybern. 80, 11 (1999).

[7] L. F. Lago-Fernández, R. Huerta, F. Corbacho, and J. A. Sigüenza, Phys. Rev. Lett. 84, 2758 (2000); H. Haken, Prog. Theor. Phys. (Suppl.) 139, 96 (2000); W. Gerstner, Phys. Rev. Lett. 76, 1755 (1996); X. Guardiola, A. DíazGuilera, M. Llas, and C. J. Pérez, Phys. Rev. E 62, 5565 (2000).

[8] W. Maass and C. M. Bishop (eds.), Pulsed Neural Networks (MIT Press, Cambridge, Massachusetts, 1998); C. Koch, Biophysics of Computation (Oxford Univ. Press, New York, 1999); D. Johnston and S. M.-S. Wu, Foundations of Cellular Neurophysiology (MIT Press, Cambridge, 1995).

[9] Gray, C. M., P. König, A. K. Engel, and W. Singer, Nature 338, 334(1989); R. Eckhorn et al. Biol. Cybern. 60 121 (1988); W. Singer, Neuron 24, 49 and 111 (1999).

[10] M. N. Shadlen and W. T. Newsome, J. Neurosci. 18, 3870 (1998).

[11] M. Timme, F. Wolf, and T. Geisel, Chaos 13, 377 (2003).

[12] G. Chartand and L. Lesniak, Graphs and Digraphs (Chapman \& Hall, London, 1996).

[13] H.J. Sommers, A. Crisanti, H. Sompolinsky, and Y. Stein, Phys. Rev. Lett. 60, 1895 (1988).

[14] For $0.01 \leq p \leq 0.2,2^{8} \leq N \leq 2^{14}$, and $\varepsilon=-0.2$, $\tau=0.05, \bar{I}=1.1$ the deviation is $\Delta r / r<0.05$.

[15] C. van Vreeswijk and H. Sompolinsky, Science 274, 1724 (1996); Neural Comp. 10, 1321 (1998). 